\title{
Factors associated with bacteraemia in febrile, non- neonatal children $<5$ years old at the paediatric outpatient clinic of the University of Port Harcourt Teaching Hospital, Nigeria
}

\author{
U C Onubogu, ${ }^{1}$ MBBS, FWACP; I C Anochie, ${ }^{2}$ MBBS, FWACP \\ ${ }^{1}$ Braithwaite Memorial Specialist Hospital, Port Harcourt, Rivers State, Nigeria \\ ${ }^{2}$ University of Port Harcourt Teaching Hospital, Port Harcourt, Rivers State, Nigeria
}

Corresponding author: U C Onubogu (utchayonubogu@yahoo.co.uk)

\begin{abstract}
Background. Fever is one of the most common presenting symptoms in the emergency room. Bacteraemia can be a cause of febrile illness in children and can have a fatal outcome if untreated. Therefore, it is important to identify factors associated with bacteraemia in febrile children in order to aid its early diagnosis and prompt treatment.

Objectives. To determine the factors associated with bacteraemia among febrile, non-neonatal, under-five children seen in the Children's Clinic of the University of Port Harcourt Teaching Hospital, Nigeria.

Methods. Febrile children aged 29 days - 59 months who presented at the outpatient clinic and whose parents gave consent were recruited between September 2010 and January 2011. Information on their age, gender, weight, symptoms, physical examination and blood culture results was collected and analysed.

Results. A total of 362 children were studied. The prevalence rate of bacteraemia was $11.5 \%$ and $22.2 \%$ in moderately and severely malnourished children, respectively $(p=0.010)$. Children with systemic inflammatory response syndrome (SIRS) had the highest bacteraemia prevalence rate $(66.7 \% ; p<0.001)$, while children with focal infection and children with fever without other symptoms or signs had bacteraemia rates of $7.1 \%$ and $3.7 \%$, respectively. There was no significant relationship between the magnitude of fever and bacteraemia $(p>0.050)$.

Conclusion. Blood cultures should be performed in febrile children who are malnourished or who have SIRS. Antibiotics should also be commenced while blood culture results are awaited.
\end{abstract}

S Afr J Child Health 2015;9(4):124-126. DOI:10.7196/SAJCH.2015.v9i4.890

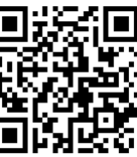

Fever is one of the most common presenting symptoms in the emergency room, accounting for $20 \%$ of paediatric emergency room visits. ${ }^{[1]}$ By 2 years of age, the average child would have had an average of 4 - 6 febrile episodes. ${ }^{[2,3]}$ Self-limiting viral illnesses have been reported to be the most common cause of fever with no other symptom or sign in young children. ${ }^{[2,4]}$ However, a febrile child with bacteraemia needs to be identified and treated in order to prevent progression to focal infection and sepsis, which can be fatal. Bacteraemia can present with fever as the only symptom; it can also present with focal infections, or as systemic infection causing sepsis. According to the International Paediatric Sepsis Consensus (IPSC) conference, a systemic inflammatory response syndrome (SIRS) resulting from an infection can be identified in the paediatric age group when two of the following criteria are met (one of which must be abnormal temperature or abnormal leukocyte count): (i) core temperature of $>38.5^{\circ} \mathrm{C}$ or $<36^{\circ} \mathrm{C}$; (ii) heart rate $>2 \mathrm{SD}$ (standard deviations) above normal for age; (iii) respiratory rate $>2 \mathrm{SD}$ above normal for age; (iv) leukocyte count elevated or depressed for age. ${ }^{[5]}$

Factors that increase the likelihood of bacteraemia in febrile children include toxicity, hyperpyrexia and malnutrition. ${ }^{[2,6-8]}$ Baraff et al. ${ }^{[2]}$ described a toxic child as one who is ill-looking, with signs of poor perfusion (cool, mottled skin or capillary refill $>2$ seconds), hypo- or hyperventilation, cyanosis and making poor eye contact. Studies have shown that children $<3$ months who are not toxic have a 1.2 - 2\% risk of bacteraemia compared with toxic infants, who have a $10-11 \%$ risk. ${ }^{[2]}$ In children between 3 and 36 months, the risk of bacteraemia is higher $(10-90 \%)$ in those who are toxic. ${ }^{[2,3]}$
The prevalence of bacteraemia correlates with the height of fever..$^{[9]}$ Children with temperatures of $39^{\circ} \mathrm{C}$ have a $4.3 \%$ chance of having bacteraemia while those with temperatures of $40^{\circ} \mathrm{C}$ have $\sim 4-17 \%$ chance of having bacteraemia. ${ }^{[9]}$ In a study involving febrile children $\leq 2$ years old who were seen in an ambulatory clinic, bacteraemia was present only if the rectal temperature was $\geq 38.9^{\circ} \mathrm{C} \cdot{ }^{[10]}$ Alpern et al.$^{[6]}$ showed that children with a temperature $\geq 40^{\circ} \mathrm{C}$ were 2.6 times more likely to have bacteraemia than if they had a lower temperature.

Immune function starts to deteriorate when weight loss exceeds $15 \% \cdot{ }^{[11]}$ Undernutrition based on protein energy malnutrition greatly increases susceptibility to major human infectious diseases in lowincome countries, particularly in children. In South Africa (SA), Reed et al. ${ }^{[7]}$ documented that the incidence of bacteraemia in malnourished children with any form of malnutrition was $9.6 \%$, $11.8 \%$ in severely malnourished children and $5.6 \%$ in stunted children. Berkowitz $^{[8]}$ also found the incidence of bacteraemia among severely malnourished SA children to be $8.9 \%$ compared with $4.8 \%$ among well-nourished children in the same study population.

Identifying factors associated with bacteraemia in febrile children will help in early diagnosis and treatment of bacteraemia, which will decrease childhood morbidity and mortality. The condition would also assist in rationalising antibiotic use, so as to prevent overprescription of broad-spectrum antibiotics.

\section{Objectives}

To determine the factors associated with bacteraemia among febrile, non-neonatal, under- 5 children in the Children's Clinic of the University of Port Harcourt Teaching Hospital (UPTH), and to determine the 
relationship between the severity of fever and bacteraemia among the study population.

\section{Methods}

Ethical clearance for the study was obtained from the Ethics Committee of the UPTH. Signed or thumb-printed written informed consent was obtained from parents/guardians of each child, after adequate explanation. A sample size of 362 was calculated using a bacteraemia prevalence rate of $38.2 \%{ }^{[12]}$ This was a prospective study done in the Children's Outpatient Clinic of UPTH from 14 September 2010 to 4 January 2011 . UPTH is a tertiary health institution located in Port Harcourt, a cosmopolitan city in southern Nigeria. The hospital serves as a referral centre for patients within and outside its locality.

Children aged 29 days - $<60$ months with axillary temperature $\geq 37.5^{\circ} \mathrm{C}$ and whose parents or guardians gave informed consent were eligible for inclusion in the study. Participants were recruited prospectively until the calculated sample size was reached. Standardised temperature and weight measurements were taken, using mercury thermometers (Hospital and Home Care LTD, UK), one infant weighing scale (Waymaster, England) and one weighing scale for older children (Ocean Med England model RGZ-160, UK). Those who had taken antibiotics or unidentified drugs within 3 days of presenting to the clinic were excluded. Participants' respiratory and heart rates were counted over 1 minute while they were at rest. Their parents or guardians were interviewed, and a physical examination was carried out on the children. Age, gender, weight, symptoms and physical examination results were entered into a standardised data collection form. Blood culture was done under sterile conditions for all the patients. Paired blood culture bottles were obtained from each patient, one for anaerobic organisms and one for aerobic organisms. All bottles were subcultured daily for up to 7 days. Colonies on the plates were identified by morphology, Gram stain and biochemical tests (coagulase, oxidase, catalase). Bacteraemia was considered positive if blood culture yielded growth of any organism within the 7 days' incubation period, except if isolates were deemed to be likely contaminants (coagulasenegative Staphylococcus and Bacillus spp.). Blood cultures were considered negative if no growth was detected during the incubation period.

Nutritional status was determined using World Health Organization growth charts, and analysed as weight-for-age $z$-scores (WAZ). Based on their clinical presentations, participants were categorised into three groups as follows: $(i)$ febrile children with no other symptoms or signs (FWS); (ii) febrile children with localised symptoms or signs (focal infection); or (iii) febrile children with SIRS (heart and respiratory rates $>2 \mathrm{SD}$ ). The data were collected and analysed using Epi Info version 3.5.1 (Centers for Disease Control and Prevention, USA). Statistical analysis was also done using the $\chi^{2}$ test and Fisher's exact test. Statistical significance was set at $p<0.05$.

\section{Results}

A total of 438 febrile children aged 29 days to $<5$ years presented to the Children's Outpatient Clinic at UPTH during clinic hours, over the 15 -week study period. Seventy-four (16.9\%) children who had taken antibiotics within 3 days of presenting to the clinic and $2(0.5 \%)$ children whose parents did not give consent were excluded, leaving 362 febrile children included in the study. There were 186 (51.4\%) males and $176(48.6 \%)$ females, and the median age was 18 (interquartile range (IQR) 9 - 28) months. A total of $285(78.7 \%)$ children had normal nutritional status (WAZ $-2-2$ ). The median WAZ was -0.28 (IQR $-1.3-0.7)$. The mean (SD) axillary

Table 1. Weight-for-age classification of children with bacteraemia

\begin{tabular}{lllll}
\hline WAZ & Negative culture, $\boldsymbol{n}(\%)$ & Positive culture, $\boldsymbol{n}(\%)$ & Total, $\boldsymbol{n}(\%)$ & $\boldsymbol{p}$-value \\
\hline $\begin{array}{l}\text { Above normal } \\
(\geq+2)\end{array}$ & $22(91.7)$ & $2(8.3)$ & $24(6.6)$ & $1.000^{*}$ \\
$\begin{array}{l}\text { Normal } \\
(>-2-<+2)\end{array}$ & $264(92.6)$ & $21(7.4)$ & $285(78.7)$ & 0.058 \\
$\begin{array}{l}\text { Moderate } \\
\text { malnutrition } \\
(-3--2)\end{array}$ & $23(88.5)$ & $3(11.5)$ & $26(7.2)$ & $0.490^{*}$ \\
$\begin{array}{l}\text { Severe } \\
\text { malnutrition } \\
(<-3)\end{array}$ & $21(77.8)$ & & & \\
$\begin{array}{l}\text { Total } \\
{ }^{*} \text { Fisher exact } p \text {-values. }\end{array}$ & $330(91.2)$ & $6(22.2)$ & $27(7.5)$ & 0.010 \\
& & $32(8.8)$ & $362(100)$ & \\
& & &
\end{tabular}

Table 2. Prevalence of bacteraemia according to clinical presentation

\begin{tabular}{|c|c|c|c|c|}
\hline $\begin{array}{l}\text { Clinical } \\
\text { presentation }\end{array}$ & Positive culture, $n(\%)$ & Negative culture, $\boldsymbol{n}(\%)$ & $\begin{array}{l}\text { Total, } n \\
(100 \%)\end{array}$ & $p$-value ${ }^{*}$ \\
\hline FWS & $1(3.7)$ & $26(96.3)$ & 27 & 0.492 \\
\hline Focal infection & $23(7.1)$ & $300(92.9)$ & 323 & 0.004 \\
\hline Diarrhoea & $4(9.3)^{\dagger}$ & $39(90.7)^{\dagger}$ & 43 & - \\
\hline Pneumonia & $2(5.3)^{\dagger}$ & $36(94.7)^{\dagger}$ & 38 & - \\
\hline URTI & $11(7.9)^{\dagger}$ & $129(92.1)^{\dagger}$ & 140 & - \\
\hline Tonsillitis & $2(4.3)^{\dagger}$ & $44(95.7)^{\dagger}$ & 46 & - \\
\hline Others & $4(7.0)^{\dagger}$ & $53(93.0)^{\dagger}$ & 57 & - \\
\hline SIRS & $8(66.7)$ & $4(33.3)$ & 12 & $<0.001$ \\
\hline Total & $32(8.8)$ & $330(91.2)$ & 362 & - \\
\hline
\end{tabular}

Table 3. Temperature pattern among children with bacteraemia

\begin{tabular}{lllll}
\hline Temperature $\left({ }^{\circ} \mathrm{C}\right)$ & $\begin{array}{l}\text { Positive culture, } \\
\boldsymbol{n}(\%)\end{array}$ & $\begin{array}{l}\text { Negative culture, } \\
\boldsymbol{n}(\%)\end{array}$ & $\begin{array}{l}\text { Total, } \boldsymbol{n} \\
(\mathbf{1 0 0 \% )}\end{array}$ & $\boldsymbol{p}$-value \\
\hline $37.5-38.0$ & $18(9.4)$ & $173(90.6)$ & 191 & 0.679 \\
$>38.0-38.5$ & $6(7.4)$ & $75(92.6)$ & 81 & 0.606 \\
$>38.5-39.0$ & $4(9.8)$ & $37(90.2)$ & 41 & $0.772^{*}$ \\
$>39.0-39.5$ & $3(9.4)$ & $29(90.6)$ & 32 & $0.754^{*}$ \\
$>39.5-40.0$ & $0(0)$ & $9(100)$ & 9 & $1.000^{*}$ \\
$>40.0$ & $1(12.5)$ & $7(87.5)$ & 8 & $0.527^{*}$ \\
Total & $32(8.8)$ & $330(91.2)$ & 362 & \\
${ }^{*}$ Fisher exact $p$-value. & & & &
\end{tabular}


temperature of the study population was $38.2^{\circ} \mathrm{C}\left(0.6^{\circ} \mathrm{C}\right)$ (range 37.5 $40.8^{\circ} \mathrm{C}$ ). Twenty-seven $(7.5 \%)$ children presented with isolated fever, 323 (89.2\%) presented with focal infection, and SIRS occurred in 12 (3.3\%). Overall, $32(8.8 \%)$ children had positive blood cultures and organisms isolated, including Staphylococcus aureus 18 (56.5\%), Pseudomonas aeruginosa 5 (15.6\%), Escherichia coli 4 (12.5\%), Klebsiella spp. 3 (9.4\%), Proteus spp. and Streptococcus spp. 1 (3.1\%). The prevalence rate of bacteraemia increased with poor nutritional status and was significantly higher $(p=0.01)$ in severely malnourished children (Table 1 ).

Children with SIRS had the highest bacteraemia prevalence rate (66.7\%), while children with FWS had the lowest (3.7\%) (Table 2). The difference in their prevalence rates was statistically significant $(p<0.001)$.

Children with temperature $>40^{\circ} \mathrm{C}$ (hyperpyrexia) had the highest prevalence of bacteraemia (12.5\%). The observed relationship between fever and presence of bacteraemia was not statistically significant (Table 3).

\section{Discussion}

The prevalence of bacteraemia in our cohort of under-5 febrile children increased significantly with decreasing weight for age, similar to findings noted by Berkowitz. ${ }^{[8]} \mathrm{He}$ reported a bacteraemia prevalence of $8.3 \%$ among severely malnourished hospitalised SA children, compared with $4.8 \%$ among their well-nourished counterparts. Reed et al. ${ }^{[7]}$ also reported a bacteraemia incidence rate of $9.6 \%$ in all malnourished children, and $11 \%$ in those with severe malnutrition in a rural SA cohort. In Kenya, Berkley et al. ${ }^{[13]}$ found that bacteraemia was significantly associated with malnutrition in children, particularly in those who were severely malnourished. It has been noted that with malnutrition, there is diminished lymphocyte, macrophage and granulocyte function. In malnutrition, there is also reduced complement activity and antibody response to infection, predisposing to infection.

The prevalence of bacteraemia in our cohort increased significantly, from a low rate $(3.7 \%)$ in those with FWS, to the highest rate $(66.7 \%)$ among those with SIRS. The low blood culture yield among FWS in this study may suggest a viral illness, which has been reported to be the most common cause of fever with no other symptoms or signs in young children. ${ }^{[2,4]}$ The $3.7 \%$ prevalence rate of bacteraemia that we observed in FWS in this study is similar to the 3\% reported by Bandyopadhyay et al. ${ }^{[14]}$ in Wisconsin. A lower prevalence rate $(1.9 \%)$ of bacteraemia among FWS was reported by Alpern et al.$^{\left[{ }^{[6]}\right.}$ in Philadelphia. The lower rate described in the latter study ${ }^{[6]}$ could be due to their exclusion of children with underlying medical illness who were more likely to have bacteraemia, unlike the present study that included such children. Children with underlying medical conditions such as HIV, renal failure, congenital heart disease and sickle cell anaemia are at higher risk of bacterial infection.

Investigators in Brazil reported that $64 \%$ of paediatric patients with SIRS have an infection. ${ }^{[15]}$ The Brazilian study differed from ours in that it was carried out in an intensive care unit where critically ill patients were more likely to be admitted; furthermore, they used at least two of the IPSC-recommended variables (clinical and/or laboratory) to make a diagnosis of SIRS. We used three clinical variables (fever, heart rate and respiratory rate) to meet the IPSC criteria for SIRS. This was done in order to maximise the use of the IPSC guidelines for diagnosing SIRS in a resource-poor setting where laboratory tests are not as freely available. It is interesting that we used the IPSC guidelines to diagnose SIRS in an outpatient clinic setting using clinical parameters that are easily available at first consultation of these patients. There is limited literature on the prevalence of sepsis in paediatric patients with SIRS as diagnosed in the outpatient setting.

In our study, children with hyperpyrexia (temperature $>40^{\circ} \mathrm{C}$ ) had the highest prevalence rate $(12.5 \%)$ of bacteraemia. However, there was no statistically significant difference in the prevalence rate when compared with those with lower temperatures. This was also found by King et al. ${ }^{[16]}$ and Yarden-Bilavsky et al., ${ }^{[17]}$ both of whom evaluated hospitalised febrile infants. In contrast, Bonadio et al.$^{[18]}$ reported that the rate of serious bacterial infection increased in direct proportion to the degree of fever, being $3.2 \%, 5.2 \%$ and $26 \%$ at temperatures of 38.1 $38.9^{\circ} \mathrm{C}, 39.0-39.9^{\circ} \mathrm{C}$ and $>40.0^{\circ} \mathrm{C}$, respectively, among febrile infants seen in an outpatient clinic in Wisconsin. However, they acknowledged that the predictive value of hyperpyrexia in identifying individual infants with serious bacterial infection is low. Stanley et al. ${ }^{[19]}$ also reported a high (38\%) prevalence of bacterial infection among children with temperatures $>40^{\circ} \mathrm{C}$ when compared with those with lower temperatures. Hyperpyrexia, especially in young infants, is known to be a risk factor for bacterial infection when assessing febrile children. ${ }^{[20]}$

\section{Conclusion}

In view of the high prevalence rate of bacteraemia among febrile, non-neonatal, under-5 children with SIRS and malnutrition, it is recommended that blood culture should be done for all such children when they are evaluated in outpatient departments. These children warrant hospitalisation and treatment with broad-spectrum antibiotic cover to treat presumed bacterial sepsis.

Acknowledgements. The authors would like to acknowledge the technical support provided by Mrs Nkereawaji Josiah, the laboratory scientist.

\section{References}

1. Graneto JW. Emergent management of pediatric patients with fever. 30 January 2014 http://emedicine.medscape.com/article/801598-overview (accessed 8 January 2015).

2. Baraff LJ, Bass JW, Fleisher GR, et al. Practice guideline for the management of infants and children 0 to 36 months of age with fever without source. Ann Emerg Med 1993;22(7):1198-1210

3. Baraff LJ. Management of infants and children 3 to 36 months of age with fever without source. Pediatr Ann 1993;22(8):497-498.

4. Jaskiewicz JA, McCarthy CA. Evaluation and management of the febrile infant 60 days of age or younger. Pediatr Ann 1993;22(8):477-480.

5. Levy MM, Fink MP, Marshall JC, et al. 2001 SCCM/ESICM/ACCP/ATS/SIS International sepsis definition conference. Crit Care Med 2003;31(4):12501256. [http://dx.doi.org/10.1097/01.CCM.0000050454.01978.3B]

6. Alpern ER, Alessandrini EA, Bell LM, Shaw KN, McGowan KL. Occult bacteraemia from a paediatric emergency department: Current prevalence, time to detection and outcome. Pediatrics 2000;106(3):505-511.

7. Reed RP, Wegerhoff FO, Rothberg AD. Bacteraemia in malnourished rural African children. Ann Trop Paediatr 1996;16(1):61-68.

8. Berkowitz F. Bacteraemia in hospitalized black South African children: A oneyear study emphasizing nosocomial bacteraemia and bacteraemia in severely malnourished children. Am J Dis Child 1984;138(6):551-556.

9. Givens TG. Fever caused by occult infections in the 3-to-36-month-old child. Pediatric Emergency Medicine Practice. EBMedicine.net 2007;4(7):1-20.

10. McCarthy PL, Dolan TF. Hyperpyrexia in children: Eight-year emergency room experience. Am J Dis Child 1976;130(8):849-851.

11. Joanna O, Zbigniew K, Małgorzata R, et al. The effect of surgical and nutritional treatment on activation parameters of peripheral blood $\mathrm{T}$ lymphocytes in stomach cancer patients in postoperative period. Pol Merkur Lekarski 2008;24(141):231-236.

12. Ayoola OO, Adeyemo AA, Osinusi K. Concurrent bacteraemia and malaria in febrile Nigerian infants. Trop Doc 2005;35(1):34-36.

13. Berkley JA, Lowe BS, Mwangi I, et al. Bacteraemia among children admitted to a rural hospital in Kenya. N Engl J Med 2005;352(1):39-47. [http://dx.doi. org/10.1056/NEJMoa040275]

14. Bandyopadhyay S, Bergholte J, Blackwell C, Friedlander J, Hennes H. Risk of serious bacterial infection in children with fever without a source in the posthaemophilus influenzae era when antibiotics are reserved for culture-proven bacteraemia. Arch Pediatr Adolesc Med 2002;156(5):512-517.

15. Carvalho PR, Feldens L, Seitz EE, Rocha T, Soledade M, Trotta A. Prevalence of systemic inflammatory syndromes at a tertiary pediatric intensive care unit. J Pediatr (Rio J) 2005;81(2):143-148.

16. King JC Jr, Berman ED, Wright PF. Evaluation of fever in infants less than 8 weeks old. South Med J 1987;80(8):948-952.

17. Yarden-Bilavsky H, Bilavsky E, Amir J, Ashkenazi S, Livni G. Relationship between fever magnitude and serious bacterial infections in febrile infants less than two-months-old. Harefuah 2009;148(11):752-755.

18. Bonadio WA, Romine K, Gyuro J. Relationship of fever magnitude to rate of serious bacterial infections in neonates. J Pediatr 1990;116(5):733-735.

19. Stanley R, Pagon Z, Bachur R. Hyperpyrexia among infants younger than 3 months. Pediatr Emerg Care 2005;21(5):291-294.

20. Lee GM, Harper MB. Risk of bacteraemia for febrile young children in the post-Haemophilus influenzae type B era. Arch Pediatr Adolesc Med 1998;152(7):624-628 